\title{
Analyzing the loading capacity of autofrettage thick cylinder with a response surface surrogate model
}

\author{
YanJun Shi ${ }^{1}$, Chenglong $\mathrm{Liu}^{1}$, XiaoJun Zheng ${ }^{2}$ \\ ${ }^{1}$ School of Mechanical Engineering, Dalian University of Technology, Dalian 116024, China; \\ ${ }^{2}$ Key Laboratory of Advanced Design and Intelligent Computing, Ministry of Education of Dalian \\ University, Dalian 116622, China;
}

Keywords: von Mises, autofrettage pressure, response surface model, optimal latin hypercube

\begin{abstract}
The paper analyzed a steel thick cylinder, established a bilinear kinematic hardening model based on the von Mises yield criterion. We also considered the parameters of the material yield limit, the autofrettage pressure, working pressure and cylinder diameters, studied of a response surface model to predict design parameters. We firstly used the optimal Latin Hypercube method to acquire the sample points. Then, we established a response surface model with these points. We also employed cross-validation error analysis for error analysis. The results tested the feasibility of this method in solving such problems.
\end{abstract}

\section{Introduction}

Autofrettage technology is a very effective method to improve the loading capacity and fatigue life of thick-walled vessel, and it's widely used in various kinds of high pressure vessel design and manufacture. The enhancement of the stress distribution inside the container can be illustrated by the following two pictures:

As we can see from the graphs above, after autofrettage treatment, the loading capacity of the cylinder will achieve a big promotion. So, studying of autofrettage pressure is of great significance. Qian Lingyun[1] proposed the optimum autofrettage pressure assessment method and provided the theory of solving process based on the working equivalent stress and circumferential stress , and they also obtained the relation curve between the maximum equivalent stress and the autofrettage pressure by the simulation results. Wang $\mathrm{Ya}$ [2] reveals the inner relationship of the important parameters of loading capacity of autofrettage vessels by analyzing the impact of super-strain, wall thickness on residual stress and elastic-plastic stress at the interface. Zhu ruilin[3] established the basic theory of autofrettage cylinder under external pressure based on fresco yield condition, and then puts forward the design and calculation algorithm. Xue Qingli[4] established the autofrettage loading and unloading for linear strain of intensive bilinear model based on the actual characteristics of autofrettage cylinder material. Autofrettage cylinder's studies were mostly based on Ansys, but traditional CAE technology is time-consuming. In recent study, the surrogate model technology shows its potential in engineering application. so, in this paper, we adopt the response surface model to analysis the problem. Yang J[5] applied response surface model to aerodynamic characteristic analysis of hypersonic aircraft. Wang Yanke[6] applied the response surface technology to the studying of the relationship between automobile suspension system and the vehicle riding comfort and handling stability, improved the efficiency. Yu Shihao[7] applied the response surface model technology to the optimization of injection molding process, greatly improved the design efficiency. Wu Xianyu[8] applied the response surface model technology to the optimization of two hypersonic inlet process, achieved significant effect . 


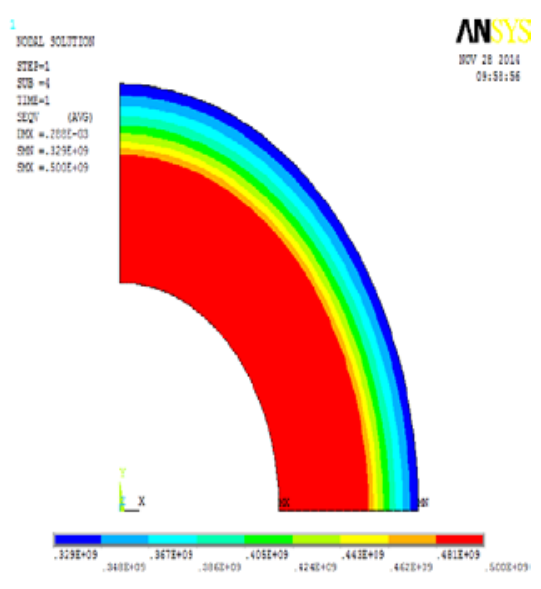

Figure.1: before processing

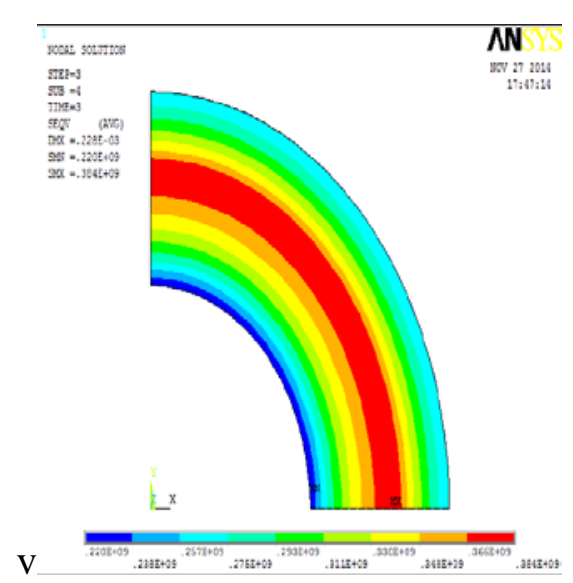

Figure.2: after processing

\section{Analysis of thick cylinder}

Autofrettage technology is by forming favorable area of residual stress in the cylinder inner surface, so as to improve the bearing capacity and fatigue life .Therefore, the value of residual stress and distribution rule is the key of autofrettage technology. the dimension of the steel thick cylinder of this example as follow:

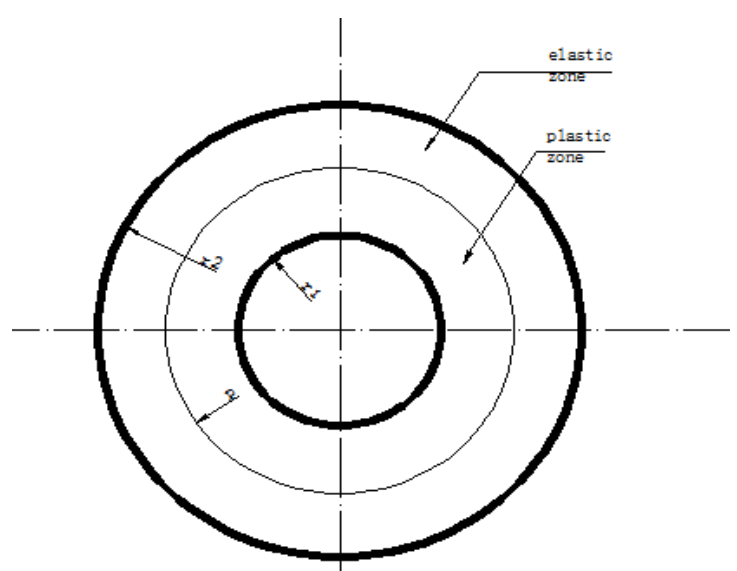

Figure.3: Thick cylinder

where, $0.09 \leq r_{1} \leq 0.11,0.04 \leq r_{2} \leq 0.06$, working pressure is 250MPa, the steel's yield limit is $500 \mathrm{MPa}(\sigma \mathrm{s})$, now, let a autofrettage pressure $\mathrm{p}(200 \mathrm{MPa} \leq \mathrm{p} \leq 500 \mathrm{MPa})$ work on the inside hole of the thick cylinder. By the theory of elastic-plastic mechanics, the inner wall of the cylinder has yielded already under the autofrettage pressure. According to the von Mises yield condition, we can get elastic-plastic interface's radius:

$$
p=\frac{2}{\sqrt{3}} \sigma_{s}\left(\ln \frac{\rho}{r_{1}}+\frac{r_{2}^{2}-\rho^{2}}{2 r_{2}^{2}}\right)
$$

After load, the stress distribution of the plastic zone for the thick wall cylinder:

$$
\begin{aligned}
& \sigma_{r}=\frac{2}{\sqrt{3}} \sigma_{s} \ln \frac{r}{r_{1}}-p \\
& \sigma_{t}=\frac{2}{\sqrt{3}} \sigma_{s}\left(\ln \frac{r}{r_{1}}+1\right)-p
\end{aligned}
$$

or and $\sigma \mathrm{t}$ represent the radial stress and tangential stress, and $\mathrm{r} 1 \leq \mathrm{r} \leq \rho$, after unloaded the pressure, the distribution of residual stress for the thick cylinder:

$$
\begin{aligned}
& \sigma_{r}=\frac{2}{\sqrt{3}} \sigma_{s} \ln \frac{r}{r_{1}}-p+\frac{p r_{1}^{2}}{r_{2}^{2}-r_{1}^{2}}\left(\frac{r_{2}^{2}}{r^{2}}-1\right) \\
& \sigma_{t}=\frac{2}{\sqrt{3}} \sigma_{s}\left(\ln \frac{r}{r_{1}}+1\right)-p-\frac{p r_{1}^{2}}{r_{2}^{2}-r_{1}^{2}}\left(\frac{r_{2}^{2}}{r^{2}}+1\right)
\end{aligned}
$$




\section{Response Surface Model}

Response surface model approximation based on polynomial fit via the least squares regression of the output parameters to the input parameters. The common order of the response surface order can be divided into linear, quadratic, cubic and quartic. It also requires a certain number of design points to be evaluated. But higher order of the response surface model may lead to over fitting for easy problems, while low order may lead to inaccuracy. so choose a suitable polynomial order is very important. This article selects quadratic function for fitting, the formula is as follows:

$$
y=a_{0}+\sum_{i=1}^{n} a_{1} x_{i}+\sum_{i=1}^{n} a_{2} x_{i}^{2}+\sum_{j<k}^{n} a_{j k} x_{j}^{l} x_{k}^{m-l} \quad, \quad 1 \leq l \leq m
$$

We choose cross-validation as the error analysis method. We select a subset of points from the main data set, remove each point at one time, re-calculate coefficients, and compare exact and approximate output values at each remove point.

\section{Design of experiment}

Sample information is the basis of constructing surrogate model. The uneven distribution of sample information in the design space will induce the precision of the surrogate model or provide a wrong model. So we should make the sample uniformly distributed within the design variable space. For complex samples, there are Latin hypercube sampling (LHS), grid sampling method, the maximum total mean square error (MSE) sampling method, the optimal Latin cube (OLHS) sampling method, etc. In order to characterize design space information sufficiently, the case choose the optimal Latin hypercube sampling plan to generate the original sampling points.

As we all known, the basic process of the Latin hypercube sampling can be describe as follows: if we want to select $m$ sample points from $n$ variables to form a $m \times n$ matrix. We firstly distribute the $n$ design variables into a range of $m$ equal length, and then select one point from each section randomly. At last, we can get $\mathrm{m}$ sample points by combine randomly. But we cannot guarantee that all these points distributed in space evenly. But the optimal Latin hypercube sampling plan will overcome this problem. In this paper, we select 300 points to construct the model.

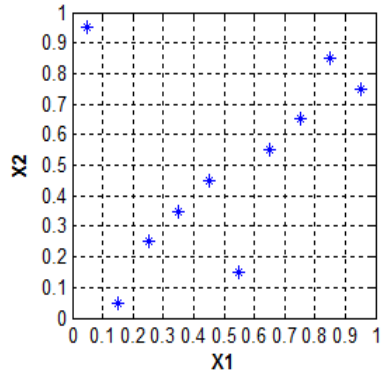

Figure.4: Latin

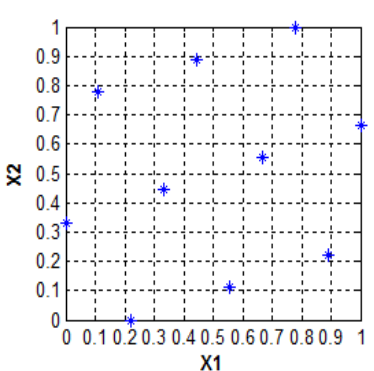

Figure.5: optimal Latin

\section{Results and Analysis}

We adapted $R^{2}$ to measure fitting degree between the approximate model and the sample points. $R^{2}=1$ means the approximate model has a high credibility. The following is the value of $\mathrm{R} 2$ and the comparison between predictive and actual value:

Table 2: fitting level of the maximum equivalent stress and the maximum strain

\begin{tabular}{ll}
\hline Parameters & $R^{2}$ \\
\hline maximum equivalent stress & 0.93805 \\
maximum strain & 0.89745 \\
\hline
\end{tabular}


Table3: predictive value and the real value

\begin{tabular}{lll}
\hline Parameters & real & \multicolumn{1}{c}{ prediction } \\
\hline emaximum equivalent stress & $3.87392 \mathrm{E} 8$ & $3.85426 \mathrm{E} 8$ \\
maximum strain & $2.46596 \mathrm{E}-4$ & $2.42364 \mathrm{E}-4$ \\
\hline
\end{tabular}

Error analysis diagrams and the optimal results are as follows:

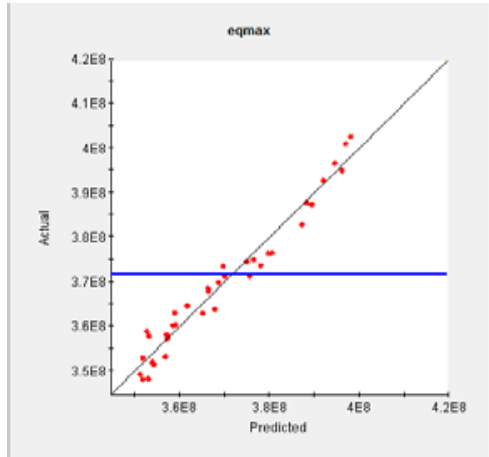

Figure.6: eqmax

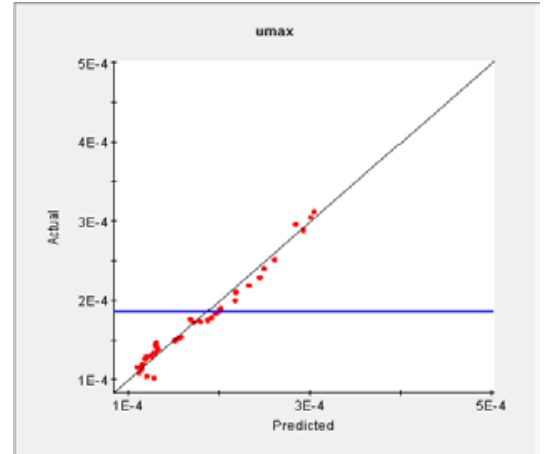

Figure.7: umax

As we can see from the two tables diagrams, we can fit the model with these sample points accurately. The predictive value can actually reflect the real value. Optimization results of autofrettage pressure is also echoed the actual engineering application

\section{Conclusions}

All the results show that respond surface model-based approximation technique show it's feasibility in solving autofrettage thick cylinder. We achieved accurate model with appropriate sample points. The optimal results not only verified the correctness of the model, and also we know how to provide an appropriate autofrettage pressure. So The availability of this technology in the field is worth studying as it can really overcome the traditional CAE's shortcomings, and also more research needs to be done with more parameters.

\section{Acknowledgement}

This work was supported by the National natural Science Foundation of China (Grand No.61304206), and the Program of Liaoning Science and Research (Grand No. L2013460).

\section{Reference}

[1]L.Y.Qian, Optimization Analysis of Autofrettage Pressure for Thick Walled Cylinders,CJME. 23(2012 )474-479.

[2] Y.Wand and R.L.Zhu, Studying of the loading capacity of the autofrettage pressure vessel ,Chemical equipment technology. 27, pp.29-34,2006.

[3] R.L.Zhu and G.L.Zhu, Design and calculation method of external pressure autofrettage cylinderl ,China mechanical engineering.15,pp.1869-1874,2010.

[4] Q.L.Xue, The theory of autofrettaged in thick-walled and it's experiments study, Pressure vessel.18,pp. 1-6,2001.

[5] J. Yang, S. P. Wu, and W. X. Hou, A method for aerodynamic characteristic analysis of hypersonic aircraft based on response surface model, Applied Mechanics and Materials Ii.477,pp. 277,2014.

[6] Y.K.Wang, Optimization design of he automobile suspension system based on response surface method,Southwest Jiaotong University:MS,2009.

[7]S.H.Yu, X.M He, G.Y. Zhang, Processing optimization of injection molding based on response surface model and NSGA-II algorithm, Journal of plasticity engineering.141,pp.5-19,2014. 
[8] X.Y.Wu, S.B.Luo, X.Q.Chen, Z.G.Wang, Response surface model based optimization of two-dimensional hypersonic inlet, Journal Acta Astronautica.pp.1127,2007. 